\title{
Fractional Flow Reserve Derived from Coronary Computed Tomography Angiography Datasets: The Next Frontier in Noninvasive Assessment of Coronary Artery Disease
}

\author{
Caroline Ball, ${ }^{1}$ Gianluca Pontone, ${ }^{2}$ and Mark Rabbat $\mathbb{D}^{1}$ \\ ${ }^{1}$ Loyola University Medical Center Department of Cardiology, Maywood, IL, USA \\ ${ }^{2}$ Centro Cardiologico Monzino, University of Milan, Italy \\ Correspondence should be addressed to Mark Rabbat; mrabbat@lumc.edu
}

Received 6 May 2018; Accepted 20 June 2018; Published 25 July 2018

Academic Editor: Ramazan Akdemir

Copyright (c) 2018 Caroline Ball et al. This is an open access article distributed under the Creative Commons Attribution License, which permits unrestricted use, distribution, and reproduction in any medium, provided the original work is properly cited.

Fractional flow reserve (FFR) derived from coronary CTA datasets $\left(\mathrm{FFR}_{\mathrm{CT}}\right)$ is a major advance in cardiovascular imaging that provides critical information to the Heart Team without exposing the patient to excessive risk. Previously, invasive FFR measurements obtained during a cardiac catheterization have been demonstrated to reduce contrast use, number of stents, and cost of care and improve outcomes. However, there are barriers to routine use of FFR in the cardiac catheterization suite. FFR $\mathrm{CT}_{\mathrm{C}}$ values are obtained using resting 3D coronary CTA images using computational fluid dynamics. Several multicenter clinical trials have demonstrated the diagnostic superiority of $\mathrm{FFR}_{\mathrm{CT}}$ over traditional coronary CTA for the diagnosis of functionally significant coronary artery disease. This review provides a background of FFR, technical aspects of $\mathrm{FFR}_{\mathrm{CT}}$, clinical applications and interpretation of $\mathrm{FFR}_{\mathrm{CT}}$ values, clinical trial data, and future directions of the technology.

\section{Introduction}

The last decade has brought rapid and exciting change to the field of cardiac imaging. In this context, coronary computed tomography angiography (CCTA) represents an excellent noninvasive tool for the evaluation of patients with coronary artery disease (CAD) [1-4]. The diagnostic accuracy of this technique has improved thanks to more effective strategies of premedication and implementation in image acquisition and postprocessing [5-10]. Likely, the most exciting technical advance is the ability to noninvasively measure the functional impact of coronary artery plaques. Advances in imaging techniques, mathematics, and computer science provide the ability to accurately measure fractional flow reserve (FFR) derived from coronary CTA datasets $\left(\mathrm{FFR}_{\mathrm{CT}}\right)$ [11]. $\mathrm{FFR}_{\mathrm{CT}}$ has the ability to provide critical information to the Heart Team without exposing the patient to unnecessary risk of an invasive procedure. What follows is a review of $\mathrm{FFR}_{\mathrm{CT}}$, including the theory and technology behind the imaging technique, accuracy data, clinical applications, and future directions.

\section{Fractional Flow Reserve: Applications}

Traditionally, coronary artery plaques were identified via invasive coronary angiography (ICA), using visual assessment of vessel stenosis to determine when a patient required revascularization, regardless of whether the visual assessment findings were supported by quantitative coronary angiographic techniques. However, oftentimes this results in revascularizing lesions that are not hemodynamically significant or lesions that are not the true etiology of the patient's symptoms, as well as failing to identify hemodynamically significant lesions [12]. Several techniques are now available in the cardiac catheterization lab to assess the hemodynamic significance of coronary lesions and therefore guide the interventional cardiologist to appropriate revascularization. At present, the most widely accepted measure of the hemodynamic significance of coronary stenoses is fractional flow reserve (FFR) which serves to identify specific vessels and lesions that are prone to induce ischemia during appropriate stress. FFR is a measure of the ratio of maximal blood flow through the coronary artery distal to a stenotic lesion to 
the normal maximal blood flow. It is traditionally measured in the cardiac catheterization lab using a pressure wire and administering an intracoronary or intravenous vasodilator to produce maximal hyperemia [13]. For example, an FFR value of 0.75 means that a stenosis is causing a $25 \%$ drop in pressure across the lesion, which means that maximal hyperemic flow is equally reduced by $25 \%$. Recent large trials have demonstrated the benefit of FFR as a tool to assess the appropriateness of revascularization, particularly for patients with stable coronary artery disease (CAD). The DEFER Trial demonstrated that it is safe to defer percutaneous coronary intervention (PCI) in patients with stable angina with lesions of $>50 \%$ visual stenosis on ICA but an invasive FFR value $\geq 0.75$ [14]. Further, the FAME I trial demonstrated that, in patients with stable multivessel CAD, using invasive FFR during PCI reduced a composite outcome of death, nonfatal myocardial infarction, and revascularization [15]. Moreover, the FAME investigators found a decreased use of contrast, fewer stents, and lower procedure-related costs in patients randomized to undergo FFR-guided revascularization. Among patients with stable CAD the FAME II trial found that PCI to lesions with an invasive FFR value $\leq 0.80$ compared with optimal medical therapy reduced the composite outcome of death, nonfatal myocardial infarction, and urgent revascularization [16]. Five-year follow-up from the FAME II trial confirmed that an FFR-guided PCI strategy was associated with a significantly lower rate of a combined outcome of death, myocardial infarction, or urgent revascularization when compared to patients managed with medical therapy alone [17]. There is a continuous, inverse relationship between the numeric FFR value and adverse outcomes, which is true regardless of whether or not the lesion is revascularized [18]. Despite its demonstrated clinical benefit and recommendations by major societies such as the American College of Cardiology (class IIa recommendation) and the European Society of Cardiology (class IA recommendation), given the invasive nature of the FFR procedure, the added time, radiation, contrast administration and cost of adenosine which must be given to patients during FFR measurement, the high costs of the pressure-sensing wires, and limited reimbursement, FFR evaluation is infrequently performed in clinical practice [1922]. Invasive FFR was performed in only $6.1 \%$ of patients using data from over 60,000 ICA cases in the American College of Cardiology registry [23]. A priori knowledge of the presence and functional significance of specific coronary artery lesions before angiography may aid the cardiologist in deciding whether or not to proceed with ICA and redefine the revascularization strategy (Figure 1).

\section{Technical Aspects of FFR $_{\mathrm{CT}}$}

Advances in computational fluid dynamics (CFD) allow determination of coronary flow from static high quality coronary CTA images. CFD is based on the Navier-Stokes equations $[24,25]$. While the Newtonian laws of motion and the understanding of viscous fluid dynamics that underpin the Navier-Stokes equations have been used in other disciplines for centuries, it was not until recent advances in supercomputing that these equations could be applied to the complex, three-dimensional, and time-sensitive flow patterns of the coronary arteries.

CFD requires defining the vessel shape anatomically, as well as adequate descriptions of the "boundary conditions" of the arterial system. To define vessel shape for CFD calculations one must first obtain coronary CTA images in accordance with the Society of Cardiovascular Computed Tomography (SCCT) guidelines to sufficiently define the vessel walls [26]. Currently, the only commercially available mechanism for computing $\mathrm{FFR}_{\mathrm{CT}}$ is via HeartFlow (HeartFlow Inc., Redwood, CA). For FFR $\mathrm{CT}_{\mathrm{T}}$, 3-dimensional (3D) geometric modelling and computationally intricate blood flow analysis require off-site supercomputing power, and boundary conditions are determined by allometric scaling laws and assumptions regarding microvascular resistance

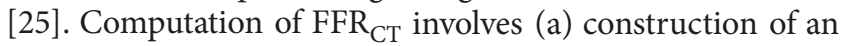
accurate patient-specific 3D anatomic model of the epicardial coronaries, (b) specifying microcirculatory models for coronary blood flow during maximal hyperemia, and (c) performing a computational solution of the laws of physics governing fluid dynamics. The physiologic model is created using the patient's anatomical model and is based on 3 scientific principles: (1) resting coronary blood flow is quantified relative to the myocardial mass. Mass can be calculated from myocardial volume, which is easily extracted from volumetric CCTA data; (2) microcirculatory resistance at rest is inversely proportional to the size of the lumen; and (3) vasodilatory response of the coronary microvasculature to adenosine is predictable. The reproducibility of $\mathrm{FFR}_{\mathrm{CT}}$ is high. In one study, the difference between the first and second FFR $_{\mathrm{CT}}$ analyses was 0.035 and for invasive FFR repeated measurements was 0.043 [27].

There is growing data evaluating the diagnostic performance of reduced order models and $1 \mathrm{D}$ processing of the image data without the use of supercomputers for coronary CTA-derived FFR [28-31]. These algorithms are not commercially available, will require more extensive testing prior to clinical use, and require approximately 1 hour of physician work effort to produce the anatomical models needed.

Advances in technology have reduced the total radiation exposure from CCTA, which results in lower radiation exposure in patients undergoing $\mathrm{FFR}_{\mathrm{CT}}$. Some centers report performing CCTA at doses $<0.1 \mathrm{mSv}$. [32]

\section{Clinical Applications and Interpretation of FFR $_{\mathrm{CT}}$ Values}

HeartFlow $\mathrm{FFR}_{\mathrm{CT}}$ has been approved by the United States Food and Drug Administration (FDA) for functional evaluation of CAD and is currently commercially available. Recently, the NICE (National Institute for Health and Care Excellence) updated their chest pain guidelines which recommend coronary CTA as the initial diagnostic test for patients with stable chest pain and suspected CAD and issued positive medical guidance on $\mathrm{FFR}_{\mathrm{CT}}$ stating the technology is safe, has high accuracy, and may avoid the need for ICA and reduce cost to the healthcare system $[33,34]$. In clinical practice, the application of $\mathrm{FFR}_{\mathrm{CT}}$ is to safely eliminate unnecessary 


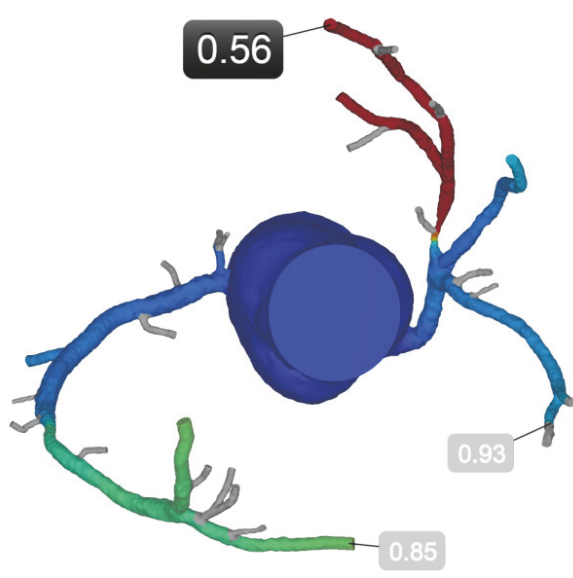

(a)

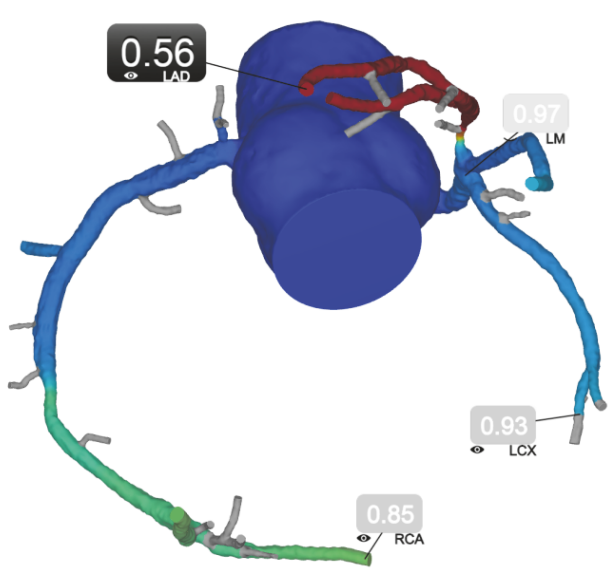

(b)

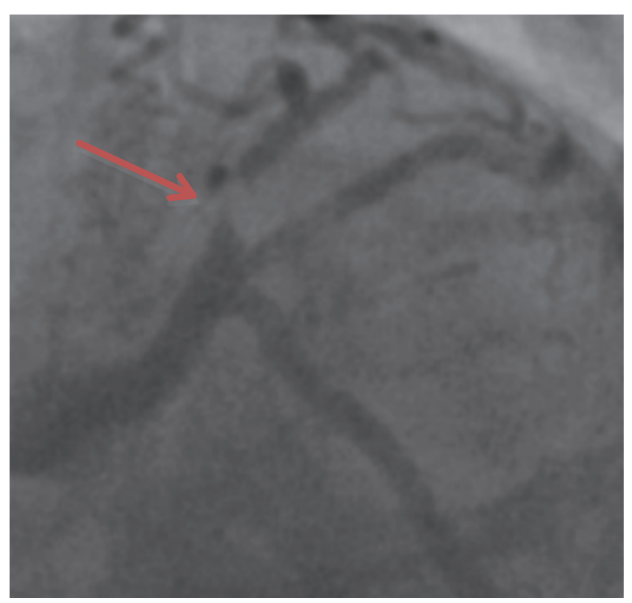

(c)

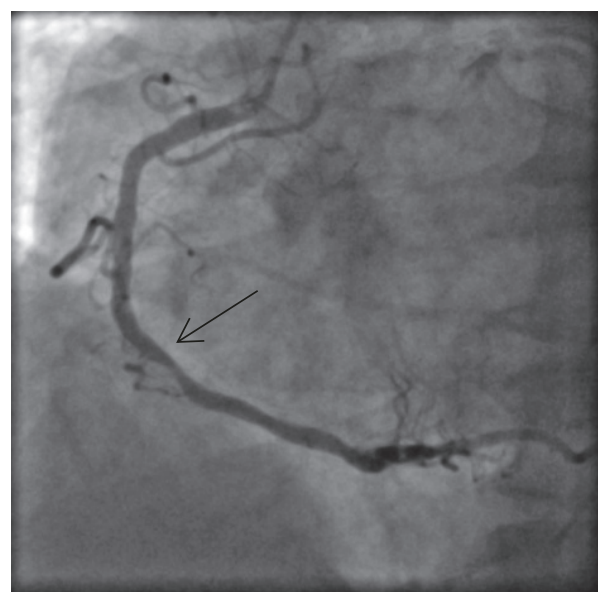

(d)

FIGURE 1: $\mathrm{FFR}_{\mathrm{CT}}$ redefining revascularization strategy. A 68-year-old male with tobacco abuse, hypertension, hyperlipidemia, diabetes, and shortness of breath underwent coronary CTA demonstrating a 70\%-90\% stenosis of the proximal LAD and a 50\%-70\% stenosis of the midRCA. The initial decision based on the coronary anatomy alone was to refer the patient for coronary artery bypass graft surgery. However, $\mathrm{FFR}_{\mathrm{CT}}$ was performed to help inform the invasive procedure. $\mathrm{FFR}_{\mathrm{CT}}$ distal to the proximal LAD and mid-RCA stenoses were 0.56 and 0.85 , respectively. The patient was rescheduled for PCI, received one stent in the proximal LAD, and is asymptomatic at three-year follow-up. Teaching points: with the functionally significant stenosis in the proximal LAD supplying a large territory of myocardium and his continued symptoms on optimal medical therapy, the patient was taken to the catheterization laboratory where a drug-eluting stent was placed. In addition, the cardiologist performed invasive FFR for the moderate stenosis in the RCA which was 0.86, corroborating the nonfunctionally significant lesion and no intervention was performed. This case highlights the unique opportunity to noninvasively provide physiological information on a per-lesion level. This enables a more informed decision around recommendations for ICA, specifically about which vessels to further interrogate and may redefine revascularization strategy. Even when the decision on referral to ICA is already taken because of symptoms and high-risk anatomy as determined by coronary CTA, FFR $_{\mathrm{CT}}$ may be of relevance by guiding decisions about other intermediate range lesions. $\operatorname{FFR}_{\mathrm{CT}}(\mathrm{a}, \mathrm{b})$ and ICA (c,d). LAD demonstrates a focal proximal severe stenosis (red arrow) that is hemodynamically significant. RCA demonstrates a focal mid moderate stenosis (black arrow) that is not hemodynamically significant. FFR $\mathrm{CT}_{\text {indicates fractional flow }}$ reserve derived from coronary computed tomography angiography (CTA) datasets; ICA, invasive coronary angiography; LAD, left anterior descending artery; and RCA, right coronary artery.

ICA and to better identify patients who may benefit from revascularization [35]. In the most recent Appropriate Use Criteria (AUC) for coronary revascularization, the American College of Cardiology recognized $\mathrm{FFR}_{\mathrm{CT}}$ as a noninvasive "combination technique" with coronary CTA to help guide treatment [36].

Currently, clinicians using $\mathrm{FFR}_{\mathrm{CT}}$ provided an interactive color-coded 3D model of the coronary tree with FFR values reported distal to stenoses [37]. The physician can manipulate the interactive model, examine each coronary segment and vessel, and determine the location and severity of lesions along the length of the coronary artery. The primary role of $\mathrm{FFR}_{\mathrm{CT}}$ both clinically and as evaluated in clinical trials is to act as an alternative to invasive FFR by evaluating the $\mathrm{FFR}_{\mathrm{CT}}$ distal to a focal stenosis. Diffuse coronary artery disease without a focal stenosis may lead to a progressive pressure drop along the length of the vessel and the treatment of these patients warrants further investigation. Nadir FFR $_{C T}$ 
TABLE 1: Summary of presented $\mathrm{FFR}_{\mathrm{CT}}$ clinical trials.

\begin{tabular}{|c|c|c|c|c|}
\hline Trial & Study Population & $\mathrm{n}$ & Intervention & Findings \\
\hline NXT & $\begin{array}{c}\text { Stable CAD } \\
\text { scheduled to undergo } \\
\text { invasive angiography }\end{array}$ & 251 & CCTA vs FFR $_{\mathrm{CT}}$ & $\begin{array}{l}\mathrm{FFR}_{\mathrm{CT}} \text { had higher diagnostic } \\
\text { accuracy than CCTA }\end{array}$ \\
\hline PLATFORM & New stable CAD & 584 & $\begin{array}{l}\text { Noninvasive stress testing } \\
\text { vs } \mathrm{FFR}_{\mathrm{CT}} \text { and ICA vs } \\
\mathrm{FFR}_{\mathrm{CT}} \text { prior to ICA }\end{array}$ & $\begin{array}{l}\text { In patients randomized to an early } \\
\text { invasive coronary angiogram for } \\
\text { stable CAD, FFR } \mathrm{CT}_{\mathrm{CT}} \text { was associated } \\
\text { with a lower rate of angiography } \\
\text { showing no obstructive CAD and } \\
\text { safe cancellation of ICA. }\end{array}$ \\
\hline RIPCORD & Stable chest pain & 200 & CTA vs $\mathrm{FFR}_{\mathrm{CT}}$ & $\begin{array}{c}\mathrm{FFR}_{\mathrm{CT}} \text { data resulted in a change in } \\
\text { management in } 36 \% \text { of cases. }\end{array}$ \\
\hline ADVANCE & Stable CAD & 1000 & CCTA Findings Reviewed & $\begin{array}{c}\text { CCTA stenosis severity, } \\
\text { importantly, even for mild CCTA } \\
\text { stenosis, in addition to diabetes and } \\
\text { hypertension were predictive of } \\
\text { abnormal FFR } \text { CT }_{\text {. }}\end{array}$ \\
\hline $\begin{array}{l}\text { Functional } \\
\text { Syntax Score }\end{array}$ & $\begin{array}{l}\text { Stable multivessel } \\
\text { disease }\end{array}$ & 77 & $\begin{array}{l}\text { Noninvasive vs invasive } \\
\text { anatomic and functional } \\
\text { SYNTAX score. }\end{array}$ & $\begin{array}{l}\text { Functional SYNTAX score utilizing } \\
\text { FFR }_{\mathrm{CT}} \text { yielded similar results to } \\
\text { those obtained invasively and } \\
\text { reclassified } 30 \% \text { of patients from the } \\
\text { high- and intermediate- SYNTAX } \\
\text { score to the low-risk tertile. } \text { FFR }_{\mathrm{CT}} \\
\text { has good accuracy in detecting } \\
\text { functionally significant lesions in } \\
\text { patients with multivessel disease. }\end{array}$ \\
\hline
\end{tabular}

$\mathrm{CAD}=$ coronary artery disease. $\mathrm{CCTA}=$ coronary computed tomography angiography. $\mathrm{FFR}_{\mathrm{CT}}=\mathrm{CTA}$-derived fractional flow reserve. $\mathrm{ICA}=$ invasive coronary angiography.

values should not be used alone when determining the need for ICA or revascularization [38]. Clinical decision-making should involve additional information such as patient history, medication use, anatomy, location of stenoses, vessel size, and suitability for revascularization. Ongoing prospective clinical registries such as ADVANCE (Assessing Diagnostic Value of Noninvasive FFR $_{\mathrm{CT}}$ in Coronary Care) will shed light on the optimal treatment strategy for patients with diffuse CAD and progressive $\mathrm{FFR}_{\mathrm{CT}}$ drop and which parameter (distal vessel tip value versus value distal to a lesion) is more appropriate to guide decision-making and yield superior prognostic information [39]. Of note, $\mathrm{FFR}_{\mathrm{CT}}$ data were analyzed in 952 of the initial 1000 patients (95.2\%) enrolled in the ADVANCE real-world registry [40].

\section{FFR $_{\text {CT }}$ Clinical Trials}

To date, several multicenter clinical trials of $\mathrm{FFR}_{\mathrm{CT}}$ have been completed and are summarized in Table 1 [41-44]. In the three large diagnostic accuracy studies comparing $\mathrm{FFR}_{\mathrm{CT}}$ and coronary CTA to invasive FFR as the reference standard, FFR $_{\mathrm{CT}}$ had better diagnostic performance than coronary CTA alone [41-43]. The NXT (Analysis of Coronary Blood Flow Using CT Angiography: Next Steps) [45] trial is the latest diagnostic performance trial of $\mathrm{FFR}_{\mathrm{CT}}$, which used the latest version 1.4 of the HeartFlow software. It was a 10-center prospective study and enrolled 254 patients and 484 vessels that were scheduled to undergo ICA for suspected stable CAD. Patients underwent coronary CTA and $\mathrm{FFR}_{\mathrm{CT}}$ prior to the planned ICA. The investigators found an increased area under receiver-operating characteristic curve for $\mathrm{FFR}_{\mathrm{CT}}(0.90,95 \% \mathrm{CI}$ 0.87-0.94) versus standard coronary CTA $(0.81,95 \%$ CI $0.76-0.87)$, which was statistically significant. Moreover, reported per-vessel sensitivities and specificities were $84 \%$ and $86 \%$, respectively [43].

The PLATFORM (Prospective Longitudinal Trial of FFRCT: Outcome and Resource Impacts) study was a large multicenter prospective clinical utility trial of $\mathrm{FFR}_{\mathrm{CT}}$ to assess clinical outcomes and sought to assess how $\mathrm{FFR}_{\mathrm{CT}}$ affects the need for ICA [44]. The PLATFORM study assigned patients with new symptoms of stable ischemic heart disease to either "usual testing" or a coronary $\mathrm{CTA} / \mathrm{FFR}_{\mathrm{CT}}$-driven strategy. For patients in the planned invasive cohort, they either went directly to ICA or were assigned to a coronary CTA/FFR $\mathrm{CT}_{\mathrm{CT}}$ strategy, with possible cancellation of the planned ICA based on the results of the coronary $\mathrm{CTA} / \mathrm{FFR}_{\mathrm{CT}}$. In the invasive arm of the PLATFORM study, a coronary CTA/FFR ${ }_{\mathrm{CT}}$ strategy resulted in cancellation of $61 \%$ of previously planned ICA without any subjects with ICA cancelled experiencing an adverse event in 1-year follow-up. The use of a combined coronary CTA and $\mathrm{FFR}_{\mathrm{CT}}$ strategy resulted in a reduction in the incidence of ICA showing nonobstructive disease by $83 \%$. Importantly, follow-up at one year demonstrated lower health care costs for those patients in the planned invasive arm who underwent $\mathrm{FFR}_{\mathrm{CT}}$ prior to ICA $[44,46,47]$. 
The FFR $_{\mathrm{CT}}$ RIPCORD study evaluated the impact of $\mathrm{FFR}_{\mathrm{CT}}$ on clinical decision-making and demonstrated that the availability of $\mathrm{FFR}_{\mathrm{CT}}$ results had a substantial effect on the labeling of significant $\mathrm{CAD}$ and management of patients compared to coronary CTA alone [48]. Data from 200 consecutive patients from the NXT trial were utilized. Three experienced cardiologists interpreted the coronary CTA data alone and reached a consensus on management strategy. $\mathrm{FFR}_{\mathrm{CT}}$ data were then revealed to the same cardiologists and a second plan for each patient was again reached by consensus. $\mathrm{FFR}_{\mathrm{CT}}$ resulted in a change in treatment decisions in $44 \%$ of patients. $30 \%$ of patients originally thought to require PCI based upon coronary CTA alone were reallocated to optimal medical therapy on the basis of a negative $\mathrm{FFR}_{\mathrm{CT}}$. In fact, $\mathrm{FFR}_{\mathrm{CT}}$ was $>0.80$ in 13 of 44 vessels $(29.5 \%)$ graded as having a stenosis $>90 \%$. In contrast, $\mathrm{FFR}_{\mathrm{CT}}$ was $\leq 0.80$ in 17 of 366 vessels (4.6\%) graded as having stenosis $\leq 50 \%$ [48]. These data and others underscore the unreliable relationship between anatomic measures of stenosis and lesion-specific ischemia $[49,50]$.

Clinical experience from Aarhus University Hospital demonstrated that deferring ICA in patients with $\mathrm{FFR}_{\mathrm{CT}}>0.80$ had favorable short-term prognosis (median follow-up period of 12 months) and was associated with a high rate of cancellation of planned ICA [51, 52].

\section{Future Directions}

The scope of $\mathrm{FFR}_{\mathrm{CT}}$ reaches far beyond the identification of FFR values [53]. New measures, such as percent myocardium at risk, are on the horizon which should further help clinicians make decisions, especially about the clinical significance of distal or branch vessel stenosis. It is conceivable that revascularization in patients with small areas of ischemic myocardium, as determined by $\mathrm{FFR}_{\mathrm{CT}}$, offers no advantage to optimal medical therapy alone. With the clinical adoption of $\mathrm{FFR}_{\mathrm{CT}}$, we are seeing individuals with diffuse atherosclerosis and/or small coronary arteries with low $\mathrm{FFR}_{\mathrm{CT}}$ values. These findings are in line with prior reports on the continuous decline in pressure along the length of diffuse atherosclerosis without focal stenosis [54]. The ratio of vascular volume to myocardial mass $(\mathrm{V} / \mathrm{M})$ may shed light into the ischemic potential of these patients and better characterize the disease states in patients with vessel sizes that are insufficient to meet myocardial demand, with or without focal stenoses [55].

Akin to the invasive arena, we may soon be able to utilize the anatomic and functional information derived from FFR $_{\text {CT }}$ to calculate SYNTAX (Synergy between PCI with Taxus and Cardiac Surgery) scores to aid clinicians to both decide between optimal medical therapy and revascularization and between PCI and coronary artery bypass graft surgery. Recently, the calculation of the noninvasive functional SYNTAX score utilizing $\mathrm{FFR}_{\mathrm{CT}}$ was noted to be feasible, yielded similar results to those obtained invasively, and reclassified $30 \%$ of patients from the high- and intermediateSYNTAX score to the low-risk tertile (REF). [56] Applications of coronary CTA-derived computational models may enable us to determine outcomes after revascularization. Virtual stenting by $\mathrm{FFR}_{\mathrm{CT}}$ demonstrated diagnostic accuracy of $96 \%$ in the prediction of residual lesions prone to ischemia when placed under the appropriate stress [57]. Virtual stenting and bypass grafting have the potential to advance our knowledge and optimize coronary revascularization. Finally, data from the EMERALD (exploring the mechanism of the plaque rupture in acute coronary syndrome [ACS] using CCTA and CFD) study illustrated that CFD derived hemodynamic forces across lesions improved the prediction of acute coronary syndrome [58]. In fact, noninvasive hemodynamic parameters were better at identifying culprit lesions causal of acute coronary syndrome than either stenosis severity or high-risk plaque features. This data demonstrates the extraordinary potential bridging CFD to coronary CTA to provide not just an FFR value but valuable insight into identifying the vulnerable patient.

\section{Conclusion}

$\mathrm{FFR}_{\mathrm{CT}}$ represents an exciting development in the evaluation of ischemic heart disease. Using advances in imaging and $\mathrm{CFD}, \mathrm{FFR}_{\mathrm{CT}}$ offers a noninvasive diagnostic strategy to identify functionally significant lesions in order to distinguish between patients who can safely avoid ICA and those patients who require revascularization.

\section{Abbreviations \\ CCTA: Coronary computed tomography angiography \\ FFR: Fractional flow reserve \\ $\mathrm{FFR}_{\mathrm{CT}}$ : Fractional flow reserve derived from coronary computed tomography angiography datasets \\ ICA: Invasive coronary angiography \\ PCI: Percutaneous coronary intervention \\ CFD: Computational fluid dynamics \\ FDA: Food and Drug Administration \\ CAD: Coronary artery disease.}

\section{Conflicts of Interest}

The authors declare no conflicts of interest.

\section{Acknowledgments}

Dr. Pontone has received institutional fees as a speaker and clinical research grants from GE and Bracco, institutional fees as a speaker from Medtronic and Bayer, and an institutional research grant from HeartFlow. Dr. Rabbat is a consultant to HeartFlow.

\section{References}

[1] D. Neglia, D. Rovai, C. Caselli et al., "Detection of Significant Coronary Artery Disease by Noninvasive Anatomical and Functional Imaging," Circulation: Cardiovascular Imaging, vol. 8, no. 3, pp. e002179-e002179, 2015.

[2] M. S. Bittencourt, E. A. Hulten, V. L. Murthy et al., "Clinical Outcomes after Evaluation of Stable Chest Pain by Coronary 
Computed Tomographic Angiography Versus Usual Care: A Meta-Analysis," Circulation: Cardiovascular Imaging, vol. 9, no. 4, 2016.

[3] A. I. Guaricci, G. Pontone, L. Fusini et al., "Additional value of inflammatory biomarkers and carotid artery disease in prediction of significant coronary artery disease as assessed by coronary computed tomography angiography," European Heart Journal - Cardiovascular Imaging, vol. 18, no. 9, pp. 1049-1056, 2017.

[4] E. Maffei, S. Seitun, K. Nieman et al., "Assessment of coronary artery disease and calcified coronary plaque burden by computed tomography in patients with and without diabetes mellitus," European Radiology, vol. 21, no. 5, pp. 944-953, 2011.

[5] A. I. Guaricci, E. Maffei, N. D. Brunetti et al., "Heart rate control with oral ivabradine in computed tomography coronary angiography: a randomized comparison of $7.5 \mathrm{mg}$ vs $5 \mathrm{mg}$ regimen," International Journal of Cardiology, vol. 168, no. 1, pp. 362-368, 2013.

[6] A. I. Guaricci, J. D. Schuijf, F. Cademartiri et al., "Incremental value and safety of oral ivabradine for heart rate reduction in computed tomography coronary angiography," International Journal of Cardiology, vol. 156, no. 1, pp. 28-33, 2012.

[7] H. Brodoefel, C. Burgstahler, I. Tsiflikas et al., "Dual-source CT: Effect of heart rate, heart rate variability, and calcification on image quality and diagnostic accuracy," Radiology, vol. 247, no. 2, pp. 346-355, 2008.

[8] N. M. Meyersohn, B. Szilveszter, P. V. Staziaki et al., "Coronary CT angiography in the emergency department utilizing second and third generation dual source CT," Journal of Cardiovascular Computed Tomography, vol. 11, no. 4, pp. 249-257, 2017.

[9] G. Pontone, E. Bertella, S. Mushtaq et al., "Coronary artery disease: diagnostic accuracy of CT coronary angiography-a comparison of high and standard spatial resolution scanning," Radiology, vol. 271, no. 3, pp. 688-694, 2014.

[10] G. Pontone, G. Muscogiuri, D. Andreini et al., "Impact of a New Adaptive Statistical Iterative Reconstruction (ASIR)-V Algorithm on Image Quality in Coronary Computed Tomography Angiography," Academic Radiology, 2018.

[11] S. H. Kueh, M. Boroditsky, and J. Leipsic, "Fractional flow reserve computed tomography in the evaluation of coronary artery disease," Cardiovascular Diagnosis and Therapy, vol. 7, no. 5, pp. 463-474, 2017.

[12] M. R. Patel, E. D. Peterson, D. Dai et al., "Low diagnostic yield of elective coronary angiography," The New England Journal of Medicine, vol. 362, no. 10, pp. 886-895, 2010.

[13] M. J. Kern MJ and M. J. Lim, "Chapter 24: Evaluation of Myocardial and Coronary Blood Flow and Metabolism," in Grossman \& Baim's Cardiac Catheterization, Angiography, and Interventions, pp. 505-544, 8th edition.

[14] G. J. W. Bech, B. de Bruyne, N. H. J. Pijls et al., "Fractional flow reserve to determine the appropriateness of angioplasty in moderate coronary stenosis: a randomized trial," Circulation, vol. 103, no. 24, pp. 2928-2934, 2001.

[15] P. A. L. Tonino, B. de Bruyne, N. H. J. Pijls et al., "Fractional flow reserve versus angiography for guiding percutaneous coronary intervention," The New England Journal of Medicine, vol. 360, no. 3, pp. 213-224, 2009.

[16] B. De Bruyne, N. H. Pijls, B. Kalesan et al., "Fractional Flow Reserve-Guided PCI versus Medical Therapy in Stable Coronary Artery Disease," The New England Journal of Medicine, vol. 367, no. 11, pp. 991-1001, 2012.
[17] P. Xaplanteris, S. Fournier, N. H. Pijls et al., "Five-Year Outcomes with PCI Guided by Fractional Flow Reserve," The New England Journal of Medicine, 2018.

[18] N. P. Johnson, K. L. Gould, M. F. Di Carli, and V. R. Taqueti, "Invasive FFR and Noninvasive CFR in the Evaluation of Ischemia," Journal of the American College of Cardiology, vol. 67, no. 23, pp. 2772-2788, 2016.

[19] N. S. Kleiman, "Bringing It All Together," Journal of the American College of Cardiology, vol. 58, no. 12, pp. 1219-1221, 2011.

[20] G. N. Levine, E. R. Bates, J. C. Blankenship et al., "2011 ACCF/AHA/SCAI Guideline for Percutaneous Coronary Intervention," Journal of the American College of Cardiology, vol. 58, no. 24, pp. e44-e122, 2011.

[21] T. Härle, U. Zeymer, M. Hochadel et al., "Real-world use of fractional flow reserve in Germany: results of the prospective ALKK coronary angiography and PCI registry," Clinical Research in Cardiology, vol. 106, no. 2, pp. 140-150, 2017.

[22] G. Montalescot, U. Sechtem, and S. Achenbach, "ESC guidelines on the management of stable coronary artery disease: the Task Force on the management of stable coronary artery disease of the European Society of Cardiology," European Heart Journal, vol. 34, no. 38, pp. 2949-3003, 2013.

[23] P. B. Dattilo, A. Prasad, E. Honeycutt, T. Y. Wang, and J. C. Messenger, "Contemporary patterns of fractional flow reserve and intravascular ultrasound use among patients undergoing percutaneous coronary intervention in the United States: Insights from the national cardiovascular data registry," Journal of the American College of Cardiology, vol. 60, no. 22, pp. 23372339, 2012.

[24] P. Rajiah and C. D. Maroules, "Myocardial ischemia testing with computed tomography: Emerging strategies," Cardiovascular Diagnosis and Therapy, vol. 7, no. 5, pp. 475-488, 2017.

[25] C. A. Taylor, T. A. Fonte, and J. K. Min, "Computational fluid dynamics applied to cardiac computed tomography for noninvasive quantification of fractional flow reserve: scientific basis," Journal of the American College of Cardiology, vol. 61, no. 22, pp. 2233-2241, 2013.

[26] S. Abbara, P. Blanke, C. D. Maroules et al., "SCCT guidelines for the performance and acquisition of coronary computed tomographic angiography: A report of the society of Cardiovascular Computed Tomography Guidelines Committee: Endorsed by the North American Society for Cardiovascular Imaging (NASCI)," Journal of Cardiovascular Computed Tomography, vol. 10, no. 6, pp. 435-449, 2016.

[27] S. Gaur, H. G. Bezerra, J. F. Lassen et al., "Fractional flow reserve derived from coronary CT angiography: Variation of repeated analyses," Journal of Cardiovascular Computed Tomography, vol. 8, no. 4, pp. 307-314, 2014.

[28] M. Renker, U. J. Schoepf, R. Wang et al., "Comparison of diagnostic value of a novel noninvasive coronary computed tomography angiography method versus standard coronary angiography for assessing fractional flow reserve," American Journal of Cardiology, vol. 114, no. 9, pp. 1303-1308, 2014.

[29] S. Baumann, R. Wang, U. J. Schoepf et al., "Coronary CT angiography-derived fractional flow reserve correlated with invasive fractional flow reserve measurements - initial experience with a novel physician-driven algorithm," European Radiology, vol. 25, no. 4, pp. 1201-1207, 2015.

[30] A. Coenen, M. M. Lubbers, A. Kurata et al., "Fractional flow reserve computed from noninvasive CT angiography data: 
Diagnostic performance of an on-site clinicianoperated computational fluid dynamics algorithm," Radiology, vol. 274, no. 3, pp. 674-683, 2015.

[31] B. S. Ko, J. D. Cameron, R. K. Munnur et al., "Noninvasive CTDerived FFR Based on Structural and Fluid Analysis," JACC: Cardiovascular Imaging, vol. 10, no. 6, pp. 663-673, 2017.

[32] F. Cademartiri, E. Maffei, T. Arcadi, O. Catalano, and M. Midiri, "CT coronary angiography at an ultra-low radiation dose $(<0.1$ $\mathrm{mSv}$ ): Feasible and viable in times of constraint on healthcare costs," European Radiology, vol. 23, no. 3, pp. 607-613, 2013.

[33] National Institute for Health and Care Excellence (NICE), "HeartFlow FFRCT for estimating fractional flow reserve from coronary CT angiography," Medical Technologies Guidance 32. Feburary, 2017. Accessed via: https://www.nice.org.uk/ guidance/mtg $32 /$ resources.

[34] National Institute for Health and Care Excellence (NICE), "Chest pain of recent onset: assessment and diagnosis," 2016. Accessed via: https://www.guidelines.co.uk/cardiovascular/ nice-chest-pain-guideline/453344.article.

[35] G. Pontone, D. Andreini, A. I. Guaricci et al., "Rationale and design of the PERFECTION (comparison between stress cardiac computed tomography PERfusion versus Fractional flow rEserve measured by Computed Tomography angiography In the evaluation of suspected cOroNary artery disease) prospective study," Journal of Cardiovascular Computed Tomography, vol. 10, no. 4, pp. 330-334, 2016.

[36] M. R. Patel, J. H. Calhoon, G. J. Dehmer et al., "ACC/AATS/ AHA/ASE/ASNC/SCAI/SCCT/STS 2017 Appropriate Use Criteria for Coronary Revascularization in Patients With Stable Ischemic Heart Disease: A Report of the American College of Cardiology Appropriate Use Criteria Task Force, American Association for Thoracic Surgery, American Heart Association, American Society of Echocardiography, American Society of Nuclear Cardiology, Society for Cardiovascular Angiography and Interventions, Society of Cardiovascular Computed Tomography, and Society of Thoracic Surgeons," Journal of the American College of Cardiology, vol. 69, no. 17, pp. 2212-2241, 2017.

[37] M. G. Rabbat, D. S. Berman, M. Kern et al., "Interpreting results of coronary computed tomography angiography-derived fractional flow reserve in clinical practice," Journal of Cardiovascular Computed Tomography, vol. 11, no. 5, pp. 383-388, 2017.

[38] S. H. Kueh, J. Mooney, M. Ohana et al., "Fractional flow reserve derived from coronary computed tomography angiography reclassification rate using value distal to lesion compared to lowest value," Journal of Cardiovascular Computed Tomography, vol. 11, no. 6, pp. 462-467, 2017.

[39] K. M. Chinnaiyan, T. Akasaka, T. Amano et al., "Rationale, design and goals of the HeartFlow assessing diagnostic value of non-invasive FFRCT in Coronary Care (ADVANCE) registry," Journal of Cardiovascular Computed Tomography, vol. 11, no. 1, pp. 62-67, 2017.

[40] H. Kitabata, J. Leipsic, M. R. Patel et al., "Incidence and predictors of lesion-specific ischemia by FFR CT : Learnings from the international ADVANCE registry," Journal of Cardiovascular Computed Tomography, vol. 12, no. 2, pp. 95-100, 2018.

[41] B. Koo, A. Erglis, J. Doh et al., "Diagnosis of Ischemia-Causing Coronary Stenoses by Noninvaive Fractional Flow Reserve Computed from Coronary Computed Tomographic Angiograms," Journal of the American College of Cardiology, vol. 58, no. 9, pp. 1989-1997, 2011.
[42] J. K. Min, J. Leipsic, M. J. Pencina et al., "Diagnostic accuracy of fractional flow reserve from anatomic CT angiography," The Journal of the American Medical Association, vol. 308, no. 12, pp. 1237-1245, 2012.

[43] B. L. Norgaard, J. Leipsic, S. Gaur et al., "Diagnostic Performance of noninvasive fractional flow reserve derived from coronary computed tomography in suspected coronary artery disease: the NXT trial (Analysis of Coronary Blood flow using CT Angiography: Next Steps," Journal of the American College of Cardiology, vol. 63, no. 12, pp. 1145-1155, 2014.

[44] P. S. Douglas, G. Pontone, M. A. Hlatky et al., "Clinical outcomes of fractional flow reserve by computed tomographic angiography-guided diagnostic strategies vs. usual care in patients with suspected coronary artery disease: The prospective longitudinal trial of FFRCT: Outcome and resource impacts study," European Heart Journal, vol. 36, no. 47, pp. 3359-3367, 2015.

[45] S. Gaur, S. Achenbach, J. Leipsic et al., "Rationale and design of the HeartFlowNXT (HeartFlow analysis of coronary blood flow using CT angiography: NeXt sTeps) study," Journal of Cardiovascular Computed Tomography, vol. 7, no. 5, pp. 279288, 2013.

[46] P. S. Douglas, B. De Bruyne, G. Pontone et al., "1-Year Outcomes of FFRCT-Guided Care in Patients with suspected coronary disease: the PLATFORM study," Journal of the American College of Cardiology, vol. 68, no. 5, pp. 435-445, 2016.

[47] M. A. Hlatky, B. De Bruyne, G. Pontone et al., "Quality-of-Life and Economic Outcomes of Assessing Fractional Flow Reserve with Computed Tomography Angiography: PLATFORM," Journal of the American College of Cardiology, vol. 66, no. 21, pp. 2315-2323, 2015.

[48] N. P. Curzen, J. Nolan, A. G. Zaman, B. L. Nørgaard, and R. Rajani, "Does the Routine Availability of CT-Derived FFR Influence Management of Patients With Stable Chest Pain Compared to CT Angiography Alone?: The FFRCT RIPCORD Study," JACC: Cardiovascular Imaging, vol. 9, no. 10, pp. 11881194, 2016.

[49] G. Toth, M. Hamilos, S. Pyxaras et al., "Evolving concepts of angiogram: fractional flow reserve discordances in 4000 coronary stenoses," European Heart Journal, vol. 35, no. 40, pp. 2831-2838, 2014.

[50] W. B. Meijboom, C. A. G. Van Mieghem, N. van Pelt et al., "Comprehensive assessment of coronary artery stenoses: computed tomography coronary angiography versus conventional coronary angiography and correlation with fractional flow reserve in patients with stable angina," Journal of the American College of Cardiology, vol. 52, no. 8, pp. 636-643, 2008.

[51] B. L. Nørgaard, J. Hjort, S. Gaur et al., "Clinical Use of Coronary CTA-Derived FFR for Decision-Making in Stable CAD," JACC: Cardiovascular Imaging, vol. 10, no. 5, pp. 541-550, 2017.

[52] J. M. Jensen, H. E. Bøtker, O. N. Mathiassen et al., "Computed tomography derived fractional flow reserve testing in stable patients with typical angina pectoris: influence on downstream rate of invasive coronary angiography," European Heart Journal - Cardiovascular Imaging, vol. 19, no. 4, pp. 405-414, 2018.

[53] G. Pontone and M. G. Rabbat, "The New Era of Computational Fluid Dynamics in CT Angiography: Far Beyond the FFR Number," JACC: Cardiovascular Imaging, vol. 10, no. 6, pp. 674676, 2017.

[54] B. De Bruyne, F. Hersbach, N. H. J. Pijls et al., "Abnormal epicardial coronary resistance in patients with diffuse atherosclerosis 
but "normal" coronary angiography," Circulation, vol. 104, no. 20, pp. 2401-2406, 2001.

[55] C. A. Taylor, S. Gaur, J. Leipsic et al., "Effect of the ratio of coronary arterial lumen volume to left ventricle myocardial mass derived from coronary CT angiography on fractional flow reserve," Journal of Cardiovascular Computed Tomography, vol. 11, no. 6, pp. 429-436, 2017.

[56] C. Collet, Y. Miyazaki, N. Ryan et al., "Fractional Flow Reserve Derived From Computed Tomographic Angiography in Patients With Multivessel CAD," Journal of the American College of Cardiology, vol. 71, no. 24, pp. 2756-2769, 2018.

[57] K. H. Kim, J. H. Doh, B. K. Koo et al., "A novel noninvasive technology for treatment planning using virtual coronary stenting and computed tomography-derived computed fractional flow reserve," JACC: Cardiovascular Interventions, vol. 7, no. 1, pp. 7278, 2014.

[58] J. M. Lee, G. Choi, B. Koo et al., "Identification of High-Risk Plaques Destined to Cause Acute Coronary Syndrome Using Coronary Computed Tomographic Angiography and Computational Fluid Dynamics," JACC: Cardiovascular Imaging, 2018. 


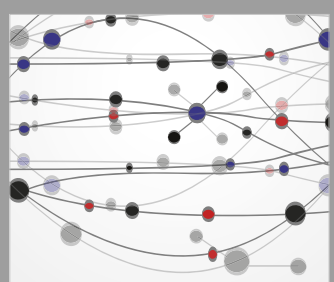

The Scientific World Journal
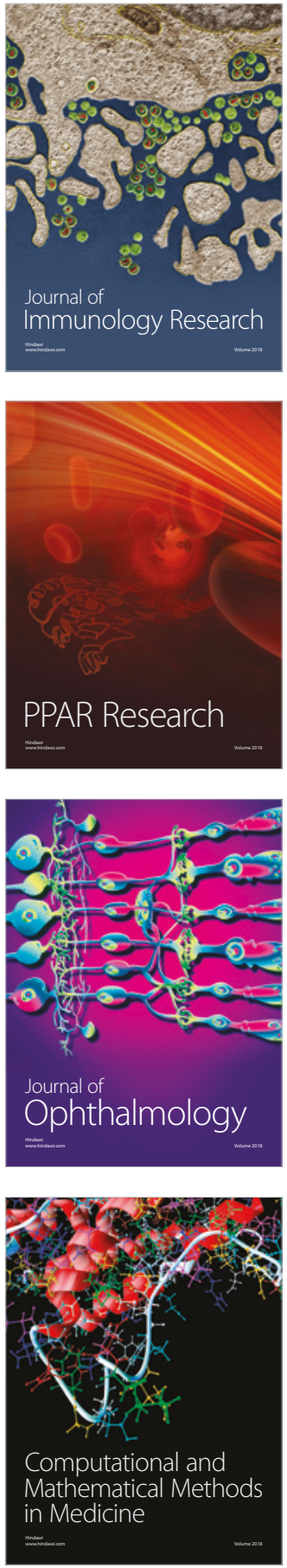

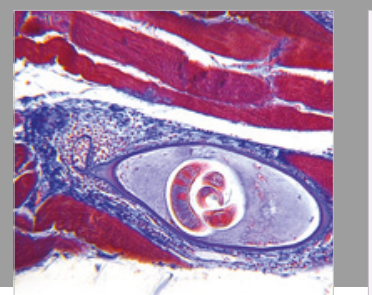

Gastroenterology Research and Practice

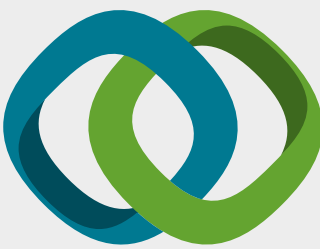

\section{Hindawi}

Submit your manuscripts at

www.hindawi.com
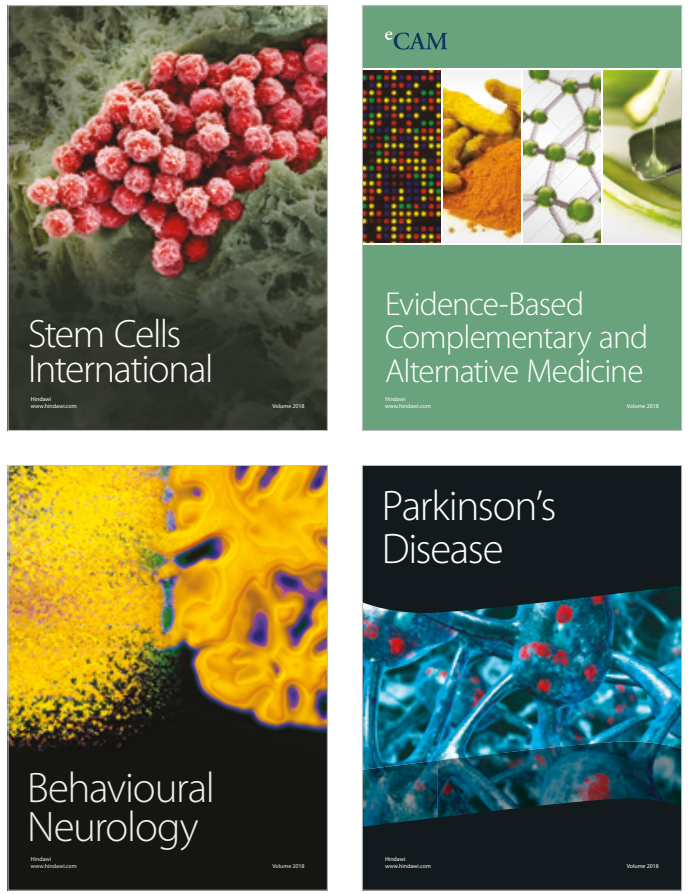

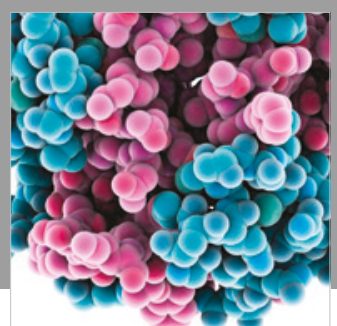

ournal of

Diabetes Research

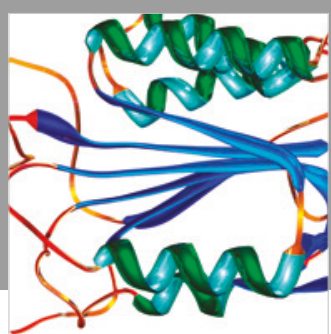

Disease Markers
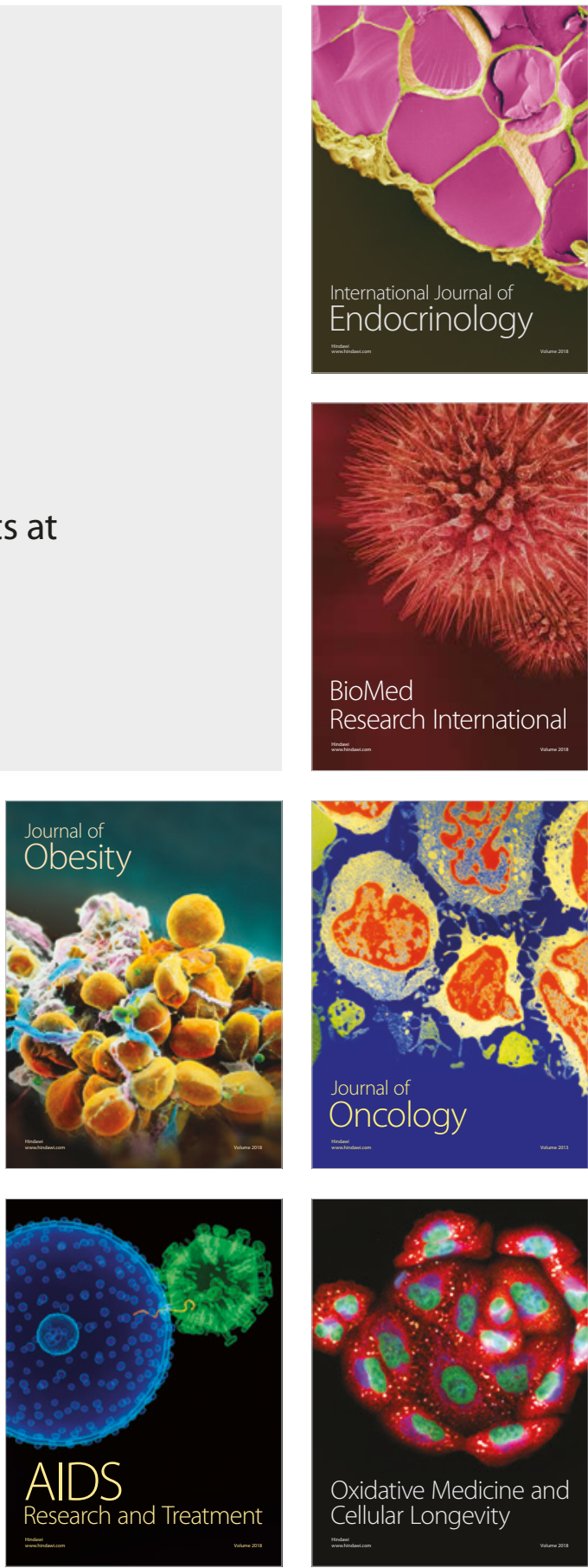